Ia

Révolution

française

\section{La Révolution française}

Cahiers de l'Institut d'histoire de la Révolution française

1 | 2012

L'attentat, objet d'histoire

\title{
Tuer le tyran ou la tyrannie? Attentat et conspiration politique : distinctions et affinités en France de 1830 à 1870
}

Murder the tyrant or kill tyranny? Political attacks and plots in France from 1830 to 1870

Jean-Noël Tardy

\section{OpenEdition}

Journals

Édition électronique

URL : http://journals.openedition.org//rf/438

DOI : $10.4000 /$ Irf.438

ISSN : 2105-2557

Éditeur

IHMC - Institut d'histoire moderne et contemporaine (UMR 8066)

Référence électronique

Jean-Noël Tardy, «Tuer le tyran ou la tyrannie ? Attentat et conspiration politique : distinctions et affinités en France de 1830 à 1870 », La Révolution française [En ligne], 1 | 2012, mis en ligne le 20 mars 2012, consulté le 02 mai 2019. URL : http://journals.openedition.org//rf/438 ; DOI : 10.4000/ Irf.438

Ce document a été généré automatiquement le 2 mai 2019.

(c) La Révolution française 


\title{
Tuer le tyran ou la tyrannie? Attentat et conspiration politique : distinctions et affinités en France de 1830 à 1870
}

\author{
Murder the tyrant or kill tyranny? Political attacks and plots in France from \\ 1830 to 1870
}

Jean-Noël Tardy

Définir l'attentat dans la France de la première moitié du XIX ${ }^{\mathrm{e}}$ siècle n'est pas chose aisée. L'attentat, c'est avant tout une qualification juridique volontairement très floue. Rien ne le prouve mieux que le débat parlementaire sur les lois de septembre 1835 sur la presse. Le gouvernement se propose alors de requalifier en «attentat à la sûreté de l'Etat », la simple offense au Roi. Les arguments des rapporteurs du projet de loi devant les chambres - il s'agit de M. Sauzet pour la Chambre des députés et du baron de Barante pour la Chambre des pairs - sont éclairants. Sauzet affirme : « Le législateur a toujours le droit, suivant les périls sociaux, d'ériger certains faits coupables en attentats; la Charte n'a pas décrété l'immutabilité de nos lois pénales ». Les orateurs opposés au projet, notamment Laboulie et Dupin dénoncent l'arbitraire du gouvernement et insistent sur la nécessaire matérialité de l'acte dans la définition de l'attentat. Contre Laboulie qui s'exclame qu' " il est évident qu'il ne saurait y avoir d'attentat sans un acte matériel, car il est l'attaque matérielle et il ne peut être autre chose », le baron de Barante et Sauzet insistent sur l'absence de définition claire de l'attentat si ce n'est par les conséquences: « une grande perturbation dans le sein de l'Etat ${ }^{1}$ ». Au XIXe siècle, le terme " attentat » en matière politique est donc un mot du pouvoir : «l'attentat » est un acte nécessairement agressif, destructeur de l'ordre public. Considéré du côté des opposants, de l'autre côté de la barricade, le même acte sera plus volontiers qualifié d'insurrection, de révolution ou de tyrannicide, des mots porteurs d'une espérance politique. 
2 C'est à la fin du XIX" siècle que se constitue l'attentat " moderne ", tel que le pratique et le théorise le courant anarchiste violent ${ }^{2}$. Il se caractérise par une volonté affichée d'ébranler les fondements d'une société donnée, au moyen d'un acte violent et volontairement spectaculaire, dans un rapport étroit à l'opinion publique. C'est toujours un défi à l'Etat et souvent un défi à la raison, tant l'écart entre le moyen employé, la cible choisie et le but recherché semble paradoxal. L'acte se rapprochant le plus de cette définition pour la période étudiée, sans en remplir tous les critères, est l'attentat contre la vie du souverain, en particulier contre celle de Louis-Philippe ${ }^{3}$. En effet, Louis-Philippe possède une descendance nombreuse et assurée. L'assassiner n'entraînera pas la fin de la dynastie et aucun des auteurs d'attentat contre sa personne ne peut se justifier par le calcul du froid Louvel ${ }^{4}$. Certaines tentatives ont coûté des vies innocentes comme l'attentat de Fieschi en 1835 ou celui d'Orsini contre Napoléon III en 1858. Les actions contre l'empereur se caractérisent par l'emploi fréquent de l'arme spectaculaire par excellence, l'explosif, et se rapprochent ainsi des pratiques du $\mathrm{XX}^{\mathrm{e}}$ siècle. Néanmoins assimiler ces actes à des attentats modernes pose problème. Les mobiles des auteurs sont souvent obscurs et le sens de leur acte est débattu avec acharnement par leurs contemporains. La plupart de ces hommes présentent d'ailleurs leur action comme un tyrannicide dont la théorisation remonte au XVI ${ }^{\mathrm{e}}$ siècle, sinon à l'Antiquité ${ }^{5}$. Mais cette rhétorique peut masquer certaines logiques moins avouables autant qu'elle éclaire le sens des évènements. L'enjeu symbolique n'échappe à aucun des acteurs et les luttes sur la légitimité de l'acte opposent les autorités aux révolutionnaires mais aussi les révolutionnaires entre eux.

3 De 1830 à 1870, la France connaît une vie politique agitée entre révolutions et coups d'Etat. Les oppositions aux régimes en place, de droite avec le légitimisme pour un court moment et surtout de gauche avec le républicanisme radical puis socialiste, ne se cantonnent pas à la lutte légale : de nombreuses sociétés secrètes apparaissent, survivent de manière presque continue sous la monarchie de Juillet mais très difficilement sous le Second Empire. Plutôt que le nombre d'attentats, c'est l'absence de toute série coordonnée qui est frappante puisque des réseaux clandestins avec les moyens nécessaires à leur réalisation existaient ${ }^{6}$. Or les attentats n'ont pas été planifiés par une autorité supérieure comme le proclame parfois un pouvoir qui fantasme l'existence de sociétés secrètes de régicides de 1830 à 1870 . Non sans succès auprès de l'opinion : après 1848 , et plus encore après 1871, «l'attentat» est associé aux groupes politiques contestant radicalement l'ordre politique et social. Le meilleur exemple de cette littérature, par ses informations privilégiées et par son ton polémique, est l'oeuvre de Maxime du Camp publiée en 1877 et intitulée significativement: Les ancêtres de la Commune: L'attentat Fieschi'. Bien sûr, ce n'est pas le corse Fieschi que vise Du Camp, ce sont les conspirateurs républicains, le prestige de combattant et martyr politique qui a pu les entourer depuis la monarchie de Juillet. Entre tous, la cible privilégiée de Du Camp est Auguste Blanqui dont l'influence est toujours vivante sous la III ${ }^{e}$ République.

4 L'objet de cette étude est donc de reprendre la question des rapports entre ceux que l'on appelle «les conspirateurs", c'est-à-dire les affiliés des sociétés secrètes engagés dans une lutte clandestine pour la République et les régicides; puis, en confrontant les renseignements tirés des archives aux discours contradictoires de la société, de retracer le conflit de représentations sur le statut politique du conspirateur du début de la monarchie de Juillet jusqu'à la chute du Second Empire, 


\title{
Conspirateurs et régicides : affinités et oppositions
}

5 Le régime de Louis-Philippe se heurte à deux oppositions antagonistes. Les partisans de la branche aînée des Bourbons, les légitimistes, qui après l'échec de la duchesse de Berry en 1832, renoncent à peu près complètement à mener une opposition violente contre le nouveau régime et les républicains qui bien que largement minoritaires, entreprennent une œuvre d'organisation et de propagande efficace dans tout le pays. Leurs sociétés politiques bénéficient de conditions favorables au début de la monarchie de Juillet. La plus célèbre d'entre elles, la Société des droits de l'homme, est semi-publique (c'est-à-dire ouverte aux seuls adhérents) jusqu'à la loi sur les associations de 1834. Si ces sociétés s'organisent en vue d'un changement de régime politique, elles ne se pensent pas uniquement comme une avant-garde mais également comme une chaîne qui doit rassembler des frères et aboutir à la constitution d'un pouvoir capable de se substituer à celui de l'Etat.

Cette conception particulière de la conspiration comme organisation d'un contre-pouvoir diffère de celle qui privilégie l'action violente d'une minorité bien préparée. Après le 13 mai 1834 où la Société des droits de l'homme s'est montrée incapable de menacer un pouvoir qui avait choisi l'épreuve de force, la première conception ne disparaît complètement même si la seconde est nettement privilégiée. A Paris, dans les sociétés secrètes des Familles puis des Saisons, où s'imposent l'ouvrier Martin-Bernard, les hommes de lettres Barbès et Blanqui qui font de la conspiration leur principale occupation, la discipline et le secret se renforcent ; la violence également, au détriment du travail de propagande. Mais leur mode d'action est l'insurrection, considérée non comme un acte de pure destruction mais comme l'affirmation de la souveraineté du peuple sur l'espace urbain. Le régicide apparaît comme un acte moins efficace, sinon moins légitime. Il s'apparente à l'assassinat pour une bonne part de l'opinion publique. Les opposants à Louis-Philippe le savent. Par ailleurs, l'attentat provoque la perte inéluctable des individus qui s'engagent à frapper le monarque: les princes et leurs proches sont entourés d'une protection qui rend presque impossible la fuite du régicide. En 1837, un placard des sociétés secrètes en voie de reconstitution (en l'occurrence, il s'agit des phalanges démocratiques qui assurent la transition entre les Familles et les Saisons) condamne les régicides :

\begin{abstract}
" À part tout ce qu'avaient de louable leurs projets, il n'y avait pas de vrais succès à en espérer, car ce n'est pas tout de tuer le tyran, il faut encore anéantir la tyrannie ; on ne pouvait et l'on ne peut encore obtenir ce DOUBLE RESULTAT qu'au moyen de l'union de tous les républicains : plus que jamais l'union fait la force.

Aussi le comité, touché de l'insuffisance ou du danger des attaques isolées, se réserve-t-il, par l'article 9 , expressément la direction des coups que la société doit porter pour atteindre le DOUBLE RÉSULTAT. Aucun sectionnaire (...) ne pourra rien tenter contre la tyrannie ou contre les tyrans, sans son ordre formel.... Couper une tête à l'hydre, c'est très-bien, mais ce serait mieux de l'écraser tout entière ${ }^{8}$ ".
\end{abstract}

7 La fascination des hommes des sociétés secrètes républicaines pour l'attentat régicide n'en est pas moins manifeste. Ils n'en ont pas l'exclusivité : l'apologie du tyrannicide est un discours commun au début de la monarchie de Juillet. Il n'est d'ailleurs pas synonyme d'abolition de la royauté. Traditionnellement, dans un cadre monarchique, le tyrannicide se conçoit comme le retour à un ordre plus ancien. Et le régicide fondateur des républicains, la mort de Louis XVI, ne prend pas la forme de l'attentat mais celui d'un 
procès. Il ne se voulait pas un fait mais un acte de droit, même si une frange des Montagnards était consciente de l'impossibilité légale de juger un roi selon les règles du système monarchiste 9 . Constatons également que les écrits les plus hostiles, les provocations au régicide sont d'abord l'œuvre de légitimistes au début des années 1830 . Elles sont dictées par leur haine personnelle contre Louis-Philippe et son père Philippe Egalité. Le royalisme d'action s'était illustré sous le Consulat par l'attentat à la machine infernale du 24 décembre 1800. Sous la monarchie de Juillet, le passage à l'acte de légitimistes reste rare : seul Bertier de Sauvigny, en fiacre, aurait cherché à écraser le roi alors qu'il traversait la rue. Mais l'influence des légitimistes sur les révolutionnaires républicains n'est pas nulle sans qu'il existe pour autant de conspiration carlorépublicaine. En 1847 plusieurs membres d'une société secrète dissidente des Nouvelles Saisons sont jugés. On leur reproche d'avoir fabriqué des engins explosifs. Plusieurs ont été arrêtés alors qu'ils se livraient à des essais barrière Saint-Denis. L'instruction affirme que la conception des projectiles a été empruntée à un groupe légitimiste arrêté en 1841. Des contacts auraient été noués entre ces hommes qui appartenaient tous à un milieu populaire. Lors des débats, un des accusés, le fils Vitou reconnaît qu'il faisait des bombes dans le but, prétend-t-il, de les vendre aux légitimistes. L'explication est peut-être fantaisiste mais on constate que l'explosif est encore associé à l'activisme royaliste, preuve de l'impact de l'attentat de la rue Saint-Nicaise dans les mémoires collectives ${ }^{10}$.

aces de cette sympathie pour les régicides parmi les hommes des sociétés secrètes sont multiples. La tentative d'un ex-soldat, Alibaud, le 25 juin 1836, suscite un réel enthousiasme dans les rangs républicains. Elle fascine car Alibaud théorise son régicide. Barbès, lui-même originaire du Sud-Ouest, ne cache pas son admiration ${ }^{11}$. Dans les sociétés secrètes lyonnaises, le pseudonyme d'Alibaud semble recherché, au point qu'il faut ajouter un chiffre après le nom ${ }^{12}$.

9 En mai 1837, un mois après le placard des Phalanges démocratiques, un journal clandestin, Le Moniteur Républicain, publie sonsixième numéro. Le journal fait l'apologie du régicide et prône cette méthode révolutionnaire à l'exclusion des autres :

« Nous regretterons qu'aux beaux jours des sociétés populaires on n'ait pas songé d'abord à s'attaquer à Louis Philippe; nous verrons dans ces oublis une preuve nouvelle de la loi de progrès; les clubs, les émeutes, les insurrections ayant manqué, l'attentat devait venir à son tour pour trancher le nœud gordien de l'avenir. C'est donc l'attentat que nous appelons de tous nos vœux, que nous entendons même exécuter tôt ou tard si personne ne nous devance. Louis-Philippe ne pouvant être traduit devant une nouvelle Convention, condamné et exécuté légalement, et l'impossibilité étant démontrée de l'attaquer en face, il ne reste pour en faire justice que le seul moyen de l'assassinat. [...] Nous concluons donc par soutenir qu'il est prématuré de s'occuper à bien discipliner les rangs démocratiques, de préparer les armes et les munitions pour le combat, qu'il n'y a qu'un seul moyen d'en finir promptement et économiquement avec la tyrannie c'est d'en abattre la tête ${ }^{13}$ ».

La proposition du Moniteur Républicain est vivement combattue par le Journal du Peuple de Dupoty dont la rédaction comprend un grand nombre d'individus initiés dans les sociétés secrètes: Dubosc, Napoléon Gallois, De La Hodde... Elle ne semble pas avoir modifié la stratégie des états-majors. Les sociétés secrètes continuent de privilégier l'insurrection à l'attentat. On constate seulement, vers 1841-1844, lorsque le successeur de Blanqui, Henri Dourille, prend la tête des Nouvelles Saisons, une ambiguïté nouvelle. Dans les ordres du jour distribués aux sectionnaires, la haine anti-nobiliaire et anti-monarchique se traduit par des phrases enflammées contre la race des Bourbons, son extinction prochaine, sans 
toutefois appeler au régicide ${ }^{14}$. Le choix d'une telle rhétorique de la part de Dourille et ses amis est réfléchi: il s'agit d'éviter ainsi la question du communisme qui divise profondément la société secrète.

Le bilan de toutes les tentatives contre Louis-Philippe ayant donné lieu à des poursuites judiciaires (13 affaires) est éclairant sur l'implication des sociétés secrètes républicaines : 9 attentats sont très certainement l'œuvre d'un seul individu ; 4 sont l'œuvre d'un groupe et relèvent de la catégorie du complot. On constate qu'un bon nombre d'attentats sont le fait d'individus en grande difficulté sociale: Meunier, employé sans avenir, souffredouleur de ses collègues, Stéphanie Girondelle finalement reconnue folle, Darmès, séparé de sa femme, en situation de grande pauvreté, Lecomte menacé de déclassement social, Henry, séparé de sa femme, au bord de la faillite et pour qui l'attentat (sans balle dans le pistolet) est une forme de suicide. Même Alibaud, ex-militaire qui ne parvient pas à trouver une situation professionnelle stable s'approche de cette catégorie. Certains ne peuvent entrer dans une société secrète et accéder au statut de conspirateur qui les fascine : c'est le cas de Fontelle et Oursel, jeunes ouvriers inconséquents, à la recherche d'une célébrité. Ils se dénoncent eux-mêmes à l'autorité ${ }^{15}$. Une majorité de tentatives est donc le fait d'individus en grande difficulté, fragilisés socialement, et en marge des groupes politiques clandestins, ignorant parfois comment entrer dans ces réseaux.

Lorsqu'il s'agit de membres de sociétés secrètes, on constate que les attentats se produisent en pleine période de désorganisation interne : après la répression d'avril 1834 pour le complot de Neuilly, combiné par des anciens affiliés de la société d'action de Kersausie $^{16}$, et pour celui de Fieschi où figurent deux affiliés de la Société des Droits de l'Homme; en 1837, où l'amnistie royale entraîne une réorganisation du monde des sociétés secrètes avec le retour de personnalités jusque-là en détention; après l'échec de l'insurrection du 12 mai 1839 avec l'affaire Darmès ${ }^{17}$. Ce sont là les actes de soldats perdus des sociétés politiques; les liens avec la structure politique centralisée se sont distendus. Il ne s'agit pas d'individus coupés du groupe social de référence, de " la classe ouvrière ", reproche célèbre adressé par Engels aux groupes blanquistes après $1871^{18}$. Dans les années 1840, le frotteur Darmès et le scieur de long Quenisset sont bien plus proches de cette classe ouvrière, de ses passions, de ses colères, de sa violence que les leaders des conspirateurs, majoritairement issus de la classe moyenne, qui cherchent alors à recréer la société des Saisons. Les régicides sont privés d'une instance politique qui peut provoquer la violence mais surtout la réguler en les empêchant de passer à l'action. La répression, menée par une autorité qui ne peut tolérer une opposition populaire organisée, favorise objectivement le passage à l'attentat.

\section{La bataille de l'opinion}

13 Le conspirateur est une figure familière de la monarchie de Juillet. Considéré depuis l'Ancien Régime comme une figure d'ennemi politique, il connaît entre la fin de la Restauration et le début des années 1830 une double réhabilitation. Une réhabilitation dans l'imaginaire politique: sur les scènes parisiennes, les conspirateurs apparaissent souvent comme des héros positifs contrairement à la tradition du mélodrame qui leur réservait des rôles de traîtres ou de mauvais conseillers du souverain. Les exemples sont nombreux et souvent inspirés de Schiller ou Byron : Fiesque renversant le doge de Gênes, Marino Faliero échouant contre le Sénat de Venise, Guillaume Tell tuant le représentant du mauvais souverain ${ }^{19}$. Le conspirateur du théâtre romantique est parfois régicide. Dans 
ce cas, le regard porté sur celui qui choisi l'assassinat politique pour faire triompher son idéal est ambigu. Les régicides à l'image d'un Hernani ou d'un Lorenzaccio sont des tempéraments aristocratiques incapables de fédérer le peuple autour de leur geste. Rares sont les dramaturges à faire l'apologie du meurtre du tyran mais ils laissent le régicide exposer son point de vue sur scène ce qui est déjà très subversif. Les doutes sur la légitimité du régicide se transforment en condamnation plus nette après l'attentat Fieschi qui entraîne par ailleurs le retour de la censure théâtrale ${ }^{20}$. A cette réhabilitation symbolique s'ajoute celle des conspirateurs bien réels de la Restauration après les Trois Glorieuses: les hommes emprisonnés pour avoir trempé dans les tentatives contre le régime des Bourbons perçoivent une indemnisation. Les conspirateurs exécutés par le précédent régime sont honorés publiquement ; il n'est pas exagéré de parler de culte des sergents de la Rochelle, tant les hommages se multiplient: monument et procession au cimetière Montparnasse, pièce de théâtre, médailles, lithographies ${ }^{21} . .$. La loi elle-même semble donner une légitimité nouvelle au conspirateur. Du moins la législation se fait moins sévère : en 1832, l'attentat à la sûreté de l'Etat est dissocié du complot. Le délit politique donne droit à un régime spécial de détention, signe d'une exception politique dans le traitement des formes de criminalité2 ${ }^{2}$.

Cette nouvelle représentation inquiète le gouvernement de Louis-Philippe qui cherche alors à affaiblir le prestige des conspirateurs, notamment en impliquant les sociétés secrètes dans les tentatives régicides. Il souhaite ainsi attribuer l'attentat Fieschi aux restes de la Société des droits de l'homme, l'attentat Meunier à la société des Familles, l'attentat Quenisset aux sociétés communistes. Le gouvernement cherche égalementà accréditer la thèse d'un lien entre ces régicides. On répand l'idée qu'une société secrète a juré la mort du Roi : ses membres désigneraient celui qui doit passer à l'acte par tirage au sort. Chacun aurait son numéro et prendrait le relais après l'échec de son prédécesseur. Cette rumeur ne s'appuie sur aucun fondement. C'est une actualisation des mythes de la secte des Assassins et de la Sainte-Vehme allemande ${ }^{23}$.

Les libéraux dénoncent le fanatisme des régicides et agitent le spectre d'un manipulateur suprême. Les références à la pièce Mahomet de Voltaire sont fréquentes. Au sujet de l'attentat Quenisset, Saint-Marc Girardin écrit ainsi qu'il «y a dans le procès de la Cour des Pairs deux choses: des doctrines et un assassinat. Selon le rapport de la Cour, les doctrines ont préparé l'assassinat. Tout se tient, et, s'il y a eu des Séides, c'est qu'il y a eu des Mahomets; Mahomets de taverne, c'est possible, mais qui savent armer leurs néophytes et les pousser au crime ${ }^{24} »$. Mais le parallèle ne prend guère car personne ne se revendique le Mahomet de ces Séides. Le pouvoir inculpe le journaliste Dupoty pour complicité morale après l'affaire Quenisset et la Cour des Pairs le condamne à dix ans de détention. Le jugement est considéré comme une atteinte à la liberté de la presse et provoque un scandale politique.

Malgré ces efforts, la politique de déconsidération des sociétés secrètes n'est guère efficace. Conspirateur et auteur d'attentat ne se confondent pas dans l'opinion sous la monarchie de Juillet. Le régicide passe pour fanatique, à la limite de la folie. Lorsqu'en 1835, les phrénologues se penchent sur le cas de Fieschi et concluent à sa normalité, et même à une certaine générosité, ils se décrédibilisent complètement ${ }^{25}$. Le conspirateur conserve une image différente, caractérisée au contraire par la rationalité, qu'elle soit malveillante ou héroïque. Les sciences ou théories qui aspirent à être reconnues comme telles font une place aux capacités du conspirateur : les phrénologues décèlent même une bosse de la conspiration. Parmi ceux-ci, Pierre-Antoine Béraud, membre lui-même des 
sociétés secrètes, est l'un des plus dithyrambiques. Il remarque que chez les conspirateurs, "le cerveau présente une saillie au-dessus et en continuation de la destructivité (...). Cette saillie est celle de la secrètivité, suivant Spurzheim, de la ruse suivant Gall ${ }^{26}$ ». Béraud, arrêté en 1839, inquiété en 1847 à Tours en même temps que Blanqui est très proche des conspirateurs néo-babouvistes. Fourier, dans Le Nouveau monde industriel et sociétaire, distingue parmi les passions fondamentales la cabaliste (la $10^{\mathrm{e}}$ passion) qu'il définit ainsi : «La cabaliste, ou esprit de parti, est la manie de l'intrigue très-ardente chez les ambitieux, les courtisans, les corporations affiliées, les commerçants, le monde galant ${ }^{27}$ ». Bien sûr, c'est loin d'être la passion la plus positive chez Fourier mais il la considère comme indispensable à l'équilibre universel du familistère.

Les sorts respectifs d'Armand Barbès après l'échec de l'insurrection de la société secrète des Saisons, le 12 mai 1839 et d'Edmond Darmès, après sa tentative ratée d'assassinat de Louis-Philippe le 15 octobre 1840 sont révélateurs des différences entre régicide et conspirateur. Les deux évènements ont été comparés d'ailleurs par les contemporains eux-mêmes. Barbès est pris blessé et les armes à la main dans les rues parisiennes. Il est traduit devant la Cour des Pairs. Il assume son acte, se défend de l'accusation d'assassinat contre un officier, le lieutenant Drouineau, et se mure dans un silence qu'il ne brise qu'occasionnellement dans quelques joutes verbales avec le Chancelier Pasquier comme lorsque l'on cherche à insinuer qu'il aurait pu tremper dans l'attentat Fieschi :

«Fieschi, monstre infernal, Fieschi, vil instrument fait pour déshonorer et pour perdre à jamais le parti politique qui l'aurait employé $2^{28} \%$. sur la personne du lieutenant Drouineau et le condamne à mort. Mais le sort de Barbès, conspirateur et combattant sur les barricades, entraîne une importante campagne d'opinion en faveur de sa grâce. Les écoles, en particulier, n'hésitent pas à prendre fait et cause pour lui. Louis Blanc rapporte que 3000 étudiants défilent jusqu'au ministère de la Justice pour demander la fin de l'échafaud politique et la grâce de Barbès ; une délégation est admise à présenter leur requête. Une seconde manifestation composée d'ouvriers est en revanche réprimée par une charge de cavalerie. Finalement Barbès obtient la grâce de Louis-Philippe comme Blanqui, arrêté quelques mois après l'échec de l'insurrection.

Un an plus tard, l'ouvrier Darmès ne bénéficie pas de la même mobilisation publique. Darmès se présente à la Cour comme "conspirateur ", autrement dit, il souhaite être reconnu comme un homme politique. Il est membre d'une des nombreuses sociétés secrètes alors en pleine division. On peut noter d'ailleurs qu'il faisait partie des manifestants qui défilaient pour la grâce de Barbès. Son comportement devant la haute cour est aussi résolu que celui du leader des Saisons. Il assume pleinement son acte et refuse de dénoncer ses complices. Pourtant, il ne fait pas l'objet du même intérêt de la part de l'opinion. Sa condition sociale, son aspect physique jouent un rôle évident. Darmès est un frotteur de parquet ; il a le front bas, la figure vulgaire. Barbès, quant à lui est un homme de lettres, par ailleurs, grand, les traits fins. Lorsque Etienne Arago le voit pour la première fois, il pense qu'il s'agit d'un jeune premier qui souhaite s'engager au Théâtre du Vaudeville ${ }^{29}$.

Il y a aussi une évidente différence de statut politique. Les voix les plus favorables à Barbès n'hésitent pas à faire des éloges dithyrambiques et à lui reconnaittre une capacité politique. Ainsi le pair de France d'Alton Shée s'insurge de la perspective d'appliquer « le châtiment du faussaire, du faux témoin, du brigand, du voleur féroce, pour Barbès! Pour 
un Français que le succès d'une révolution parisienne, en mai 1839, eût installé très naturellement au conseil d'état de la république, provisoire ou définitive, sous je ne sais quels chefs, civils ou militaires ${ }^{30} »$. Tout autre est le traitement de Darmès; une des rares voix audibles à lui être favorable, son avocat, demande son internement pour folie plutôt que la mort car il juge qu'un simple ouvrier n'avait rien à gagner de la mort de LouisPhilippe ${ }^{31}$. Darmès est finalement exécuté. A la différence sociale entre ces deux hommes s'est ajoutée la différence morale de leurs actes respectifs qui accable Darmès aux yeux de l'opinion bourgeoise. Barbès est considéré comme un « conspirateur » comme le membre d'une opposition clandestine, destinée à occuper des fonctions éminentes en cas de changement de pouvoir. Darmès est sans cesse ramené à la figure de l'assassin régicide.

\section{Vers une assimilation de la conspiration à l'attentat}

21 Après février 1848, la figure du conspirateur connaît un bref moment de gloire. Mais cette mode n'est en rien comparable à celle des années 1830-1832. Les conspirateurs de la monarchie de Juillet, divisés entre ceux qui maintiennent l'ordre avec Caussidière et Ledru-Rollin, et ceux qui souhaitent accélérer la marche des réformes dans les clubs de Paris et des grandes villes de province, excitent une peur sociale grandissante. La conspiration est également délégitimée, y compris par ceux qui l'incarnaient autrefois, par l'irruption du suffrage universel dans la vie politique. Le 15 mai 1848 est un tournant : les clubs en envahissant l'Assemblée se sont rendus coupables d'un « attentat » contre la représentation nationale. Cette journée rocambolesque passe pour la preuve de l'aliénation mentale des conspirateurs ${ }^{32}$. «J'ai toujours pensé que dans les révolutions et surtout dans les révolutions démocratiques, les fous, non pas ceux auxquels on donne ce nom par métaphore, mais les véritables, ont joué un rôle politique très considérable " écrit Tocqueville dans ses Souvenirs, en guise de conclusion au récit de cette journée. On peut lire la description de Tocqueville et sa conversation avec Ulysse Trélat comme un bon exemple de lecture médicale de la politique ${ }^{33}$.

On suppose aux hommes de l'extrême gauche des projets de plus en plus destructeurs, véritablement terroristes (le terme est utilisé en référence à 1793) : il s'agit ni plus ni moins que de brûler Paris ou Lyon. La psychose atteint son paroxysme durant l'insurrection de juin 1848 à Paris. On publie des mémoires de conspirateurs repentis comme Chenu ou d'indicateurs comme La Hodde ${ }^{34}$. L'action des membres des sociétés secrètes de la fin de la monarchie de Juillet est présentée sous le jour le plus violent. La fin de l'année 1848 signe le début d'un processus de répression de grande ampleur qui culmine après le coup d'état du 2 décembre 1851. L'opposition républicaine prend le chemin de l'exil ou entre dans une clandestinité de plus en plus périlleuse sur le territoire 35 .

Sous le Second Empire, la tentation du régicide est forte contre celui qui cultive le culte de Jules César. La succession du trône impérial est la grande faiblesse du régime. Les tentatives d'assassinat contre Napoléon III se multiplient alors. Dans la mémoire collective, on se souvient plus particulièrement des attentats italiens : Pianori (qui a tiré sur Napoléon III le 28 avril 1855), Tibaldi (avril 1857), Orsini (attentat à la bombe du 14 janvier 1858, 8 morts, 50 blessés) et Greco (janvier 1864). Les Italiens ne sont pas tout à fait des tyrannicides. Nous sommes en présence d'un autre type de violence politique. Lorsqu'ils présentent leur défense, ils s'affirment comme des patriotes. En éliminant Napoléon III, ils espèrent se venger de celui qui a détruit la République romaine en 1849, 
et par la même occasion changer le système international. Comme le fait remarquer un républicain français après la chute de l'Empire : «Il s'agit, pour eux, de renverser le joug d'un despotisme extérieur, non de changer une forme gouvernementale, non enfin de faire triompher tel système politique contre un autre ${ }^{36} »$.

On accuse, pas toujours à tort, Mazzini d'animer dans l'ombre, ces tentatives ${ }^{37}$ : Mazzini est un conspirateur emblématique depuis la monarchie de Juillet et son bref passage au pouvoir à Rome en 1849. Il passe pour avoir justifié la politique du poignard dans une réponse à Manin qui le mettait en cause dans le Times, en juillet $1856^{38}$. En fait il ne justifie l'assassinat politique que s'il permet à la révolution d'éclater. Mais les tentatives italiennes brouillent les frontières entre l'assassinat politique et les anciennes conceptions de la conspiration. Dans l'opinion, Mazzini devient le Vieux de la Montagne à la tête d'une nouvelle secte d'assassins. L'image apparait sous la plume de Crétineau-Joly, l'historien légitimiste et ultramontain, en 1850. Elle est reprise par des hommes de lettres moins conservateurs tel le bonapartiste Amédée Gabourd; une partie des socialistes français les rejoignent dans leur hostilité au leader italien, même si leur critique vise plus sa posture religieuse que sa violence politique. La presse diffuse ce qui devient vite un poncif, s'appuyant sur une italianophobie traditionnelle qui associe l'Italien à la conspiration, au poignard et à l'assassinat ${ }^{39}$.

Des Français tentent également d'assassiner l'Empereur. Le complot de l'hippodrome ou de l'Opéra comique est le plus important par le nombre des conjurés. Il prévoit l'assassinat de Napoléon III suivi d'une insurrection ouvrière dans la capitale. L'affaire, connue très tôt de la police, échoue en 1853. Parmi les conjurés on retrouve l'étudiant Ranc et le jeune Jules Vallès, relâché faute de preuves. Vallès dans un chapitre du Bachelier restitue admirablement «la fièvre du régicide» qui le tenait à cette époque ${ }^{40}$. Une tentative d'attentat contre le train de l'Empereur est déjouée en 1855 entre Pérenchies et Lambersart. Elle est élaborée par les frères Jacquin et un réseau républicain du Nord de la France, déjà entrevu dans l'affaire précédente ${ }^{41}$.

Il y a parfois coopération entre Français et Italiens : l'attaque à la bombe d'Orsini est en fait préparée depuis Londres et Bruxelles par un réfugié français, Simon Bernard dit Bernard le clubiste $^{42}$. L'examen des pièces du dossier laisse deviner moins une organisation qu'un cercle de connaissances et d'amis prêts à apporter une aide à l'entreprise : argent, conservation des bombes, transport vers Paris. Ces hommes ne sont pas des affiliés, ils n'ont pas prêté serment ${ }^{43}$. Lors du procès en Angleterre, ils affirment tout ignorer de l'entreprise. Les exilés qui souhaitent lutter par la violence contre Napoléon III sont incapables d'organiser des réseaux clandestins comparables aux sociétés secrètes de la Restauration et de la monarchie de Juillet. Le démantèlement de la Marianne dans l'Ouest entre 1854 et 1856 met un terme à leurs espérances. Le tyrannicide apparaît plus en rapport avec leur capacité limitée. Toutefois un mouvement clandestin refuse l'attentat régicide: les blanquistes. Blanqui dénonce avec constance la vanité du geste et concentre tous ses efforts en vue d'une prise d'armes à Paris. En prison, constatant l'affaiblissement du parti démocratique, il écrit à son lieutenant Rougée en exil à Londres :

«Il n'est pas jusqu'à la pensée du Régicide que vous me dites avoir surgi dans tous les esprits qui ne vienne confirmer ce triste pronostic. C'est une pensée de désespoir, le dernier recours des vaincus qui sentent les chances leur échapper et qui ont perdu toute perspective de revanche. On s'était cru jusqu'ici trop sûr de l'avenir pour se jetter dans cette voie extrême. La certitude de l'emporter de haute lutte ne permettait pas à l'idée césaricide de se faire jour. Mais si réellement elle 
s'est emparée de toutes les têtes, elle accuse alors un sentiment universel et irrésistible d'impuissance ${ }^{44} »$. parisien. Ils cherchent ailleurs une puissance à opposer à l'appareil d'Etat. Ainsi les régicides gagnent en technicité. Les conspirateurs en exil compensent leur faiblesse par la puissance technologique. Les «alchimistes de la Révolution » deviennent de véritables techniciens ${ }^{45}$. Le fulminate de mercure entre dans l'arsenal des activistes politiques. L'explosif est connu depuis 1800 mais sa méthode de fabrication ne se diffuse réellement qu'après 1848. On l'obtient grâce à un mélange d'alcool, d'acide nitrique et de mercure. C'est en Belgique que les proscrits l'expérimentent: en 1854, à la demande du réfugié français H. Magen, le fabriquant d'armes de Liège, Sanders, met au point des bombes au fulminate de mercure ${ }^{46}$. Les projets de Magen n'ont pas de commencement d'exécution mais les explosifs saisis sont exposés et inspirent sans nul doute les frères Jacquin puis Simon Bernard. Les frères Jacquin utilisent une charge explosive au fulminate de mercure reliée par un fil à une pile Bunzen pour provoquer l'explosion du train de l'Empereur à 35 mètres de distance. La charge explosive est découverte avant le voyage officiel. Par la suite, Simon Bernard perfectionne les bombes de Sanders pour Orsini.

Dans une partie de l'opinion, la distinction entre l'insurrection et l'assassinat politique perd de sa signification, les deux pratiques faisant l'objet d'une réprobation semblable : « La logique qui admet le fusil admet implicitement le poignard ; si elle exclut le poignard, elle exclut le fusil » écrit Emile de Girardin, au sujet de la controverse Manin-Mazzini ${ }^{47}$. Ce qui renforce encore cet effacement de la frontière entre conspirateurs et néo-régicides, c'est la réévaluation de l'image des auteurs d'attentat à l'explosif, pourtant susceptible de frapper des innocents. En 1835, Fieschi était considéré comme un monstre; certes il pouvait être l'objet d'une fascination trouble, à l'image d'un Lacenaire, mais son acte était considéré comme antipolitique, presque apolitique dans sa monstruosité. Les concepteurs des nouvelles "machines infernales », les ingénieurs Jacquin et le chimiste Simon Bernard trouvent des protecteurs, voire des justificateurs. Ainsi ne sont-ils pas extradés vers la France. La Cour de cassation belge estime que ce qui est reproché aux frères Jacquin est de l'ordre du crime politique et les met à l'abri de la justice française. Ce n'est qu'après cette décision qui fait scandale en France qu'un traité entre la France et la Belgique est passé pour qu'à l'avenir les inculpés de tentative d'assassinat contre la personne d'un chef d'Etat puissent être extradés. En revanche en Angleterre, Simon Bernard est jugé et acquitté par le jury dans un climat de liesse populaire à Londres. Le gouvernement n'est pas en mesure d'imposer un accord similaire à celui de la France et de la Belgique.

30 Et si beaucoup de réfugiés prennent leur distance avec la violence politique des auteurs d'attentat, certains les approuvent pleinement : c'est le cas de Félix Pyat dont les écrits extrêmement violents, sont instrumentalisés par le pouvoir impérial ${ }^{48}$. Félix Pyat, dramaturge et conspirateur, a mis en scène de nombreux conjurés romantiques entre 1830 et $1848^{49}$. Analysant et justifiant Orsini, il salue le héros et le martyr. Il se démarque seulement du moyen. Assimilant l'attentat à la conspiration, Pyat précise : 
«Si nos moyens diffèrent, notre cause est la même. Nous espérons que votre exemple ne sera pas perdu pour nous dans la mesure de nos aptitudes et de nos forces. Chacun les siennes. Si nous n'avons pas la vertu de la conspiration, nous avons parfois celle de l'insurrection qui n'est après tout que la conspiration collective $\mathrm{e}^{50} »$.

31 Félix Pyat fait la jonction entre le conspirateur héroïque de la monarchie de Juillet et l'activiste du Second Empire.

Enfin plus étonnant, Orsini a failli être gracié. A l'audience et avec l'accord de Napoléon III lui-même, l'avocat d'Orsini Jules Favre lit une lettre à l'Empereur dans laquelle son client supplie le chef de l'Etat français d'intervenir en faveur de sa patrie. Cette opération peut-être montée par l'Empereur lui-même, bouleverse la représentation de l'évènement et le sens du procès. A contre-courant de la presse et de la majorité de ses soutiens, l'Empereur reconnaît la justesse de la cause d'Orsini et évite de faire l'amalgame entre l'auteur de l'attentat et le criminel de droit commun. La justification nouvelle d'Orsini ne répond plus à la logique du tyrannicide. Le tyrannicide a pour objectif de faire cesser la tyrannie en supprimant la personne du tyran. Il est sa propre fin. Orsini en donnant une nouvelle interprétation à son acte - interpeller la France sur le sort de l'Italie transforme l'attentat à la bombe en opération médiatique d'une résonance inédite. Il s'attire ainsi des sympathies y compris auprès de ses victimes, l'impératrice et le préfet de police Piétri.

Néanmoins, il faut se garder de surestimer ce mouvement de sympathie dans l'opinion française. D’après les informations dont l'on dispose par les rapports des préfets et des procureurs généraux, l'acte est impopulaire car il a fait des victimes collatérales. Orsini n'est pas une figure politique très admirée, en partie parce qu'il est italien. Au moment de l'entrée en guerre contre l'Autriche, les procureurs généraux signalent dans leur rapport leur crainte de troubles dans les départements à cause du sentiment d'hostilité envers les Italiens, assimilés à des assassins politiques et des manipulateurs ${ }^{51}$.

Ainsi le Second Empire, en réprimant les sociétés secrètes sur le territoire français, a-t-il profondément reconfiguré les formes de la participation mais aussi de la violence politique. Aux conspirateurs des sociétés secrètes qui cherchaient à contrôler la violence pour s'organiser comme un Etat dans l'Etat, a succédé le conspirateur exilé de sa patrie, cherchant par l'attentat à venger le crime du 2 décembre, voire à bouleverser l'ordre européen. De toutes les tentatives contre Napoléon III, celle d'Orsini et de ses complices est la plus complexe. D'une violence peu commune, elle était le fruit d'aspirations multiples coalisées pour l'occasion. Cela explique en partie la multiplicité des interprétations : présentée comme un tyrannicide par certains Français, comme un acte de guerre par les Italiens, elle change une dernière fois de sens au procès et devient un appel à l'aide en faveur de l'Italie. Les victimes tendent à être mises au second plan, considérées comme partie négligeable comparé aux enjeux politiques, ou assimilées à des soutiens de l'Empire, comme si le suffrage universel avait fait de tous les individus, en même temps que des acteurs politiques, des victimes légitimes pour les auteurs d'attentat. Après le 2 décembre, la Semaine sanglante fait franchir à la terreur étatique un nouveau stade. L'exécution massive de communards alors que les combats ont cessé, l'emploi dans certains cas de la mitrailleuse, dépasse dans l'horreur la répression de juin 1848 et marque durablement l'extrême gauche révolutionnaire. Comme le fait remarquer le journaliste du Figaro Albert Wolff, le conspirateur romantique disparait pour laisser place à l'anarchiste ${ }^{52}$. Avec les vagues d'attentats de la fin du siècle, commence le temps 
de l'attentat moderne, même si on peut légitimement se demander jusqu'à quel point l'opinion n'était pas déjà préparée à ce type d'actes.

\section{NOTES}

1. Le débat est reproduit dans Jean-Baptiste. DUVERGIER, Collection complète des lois, décrets, ordonnances, règlemens et avis du conseil d'Etat, Paris, Guyot et Scribe, 1836, p. 259-260.

2. La bibliographie sur le sujet est considérable. Par commodité on renverra à celle de la fin de l'ouvrage de John MERriman, Dynamite club. L'invention du terrorisme à Paris, Paris, Tallandier, 2009, p. 247-255.

3. Les tentatives de régicide qui nous intéressent ici ont fait l'objet d'une thèse : Karelle VINCENT, Le Régicide, de Saint Réjant à Gorguloff (1800-1932). Perception et représentations, thèse pour le doctorat d'histoire de l'Université de Bourgogne sous la direction de M. Jean-Marc Berlière, 2000 ; voir aussi la synthèse quelque peu apologétique mais bien documentée de Laurent LOUESSARD, L'épopée des régicides. Passions et drames 1814-1848, Paris, Cahors, Soupir L'Insomniaque, 2000, le chapitre de l'ouvrage de Jill HARSIN, Barricades. The war of the Streets in Revolutionary Paris, 1830-1848, New York: Palgrave/Macmillan, 2002, p. 147-210et surtout désormais l'ouvrage de Karine SALOMÉ, L'Ouragan homicide. L'attentat politique en France au XIX ${ }^{e}$ siècle, Seyssel, Champ Vallon, 2010.

4. En 1820, le sellier Louvel assassine le duc de Berry car c'est le seul héritier mâle de la branche aînée des Bourbons capable d'avoir une descendance. Sa mort devait entraîner l'extinction de la dynastie. La naissance d'un héritier, «l'enfant du miracle » peu après la mort du duc déjoua les espérances du régicide. Voir Gilles MALANDAIN, L'introuvable complot. Attentat, enquête et rumeur dans la France de la Restauration, Paris, Editions de l'EHESS, 2011.

5. Franklin L. FORD, Le Meurtre Politique, du tyrannicide au terrorisme, Paris, PUF, 1990 ; David GEORGE «Distinguishing Classical Tyrannicide from Modern Terrorism », The Review of Politics, vol. 50, $\mathrm{n}$ - 3, été 1988, p. 390-419.

6. La société secrète des Saisons achetait de la poudre ou en fabriquait artisanalement. Elle aurait pu l'utiliser comme explosif pour un attentat mais a préféré en réserver l'usage pour faire des munitions. Au 12 mai 1839, Blanqui affirme que l'arsenal des Saisons comprenait 10000 cartouches. Louis-Auguste BLANQUI, "Réponse au document Taschereau», dans Théophile Silvestre, L.-A. Blanqui, étude historique, Paris, Poulet-Malassis, 1862, p. 361.

7. Maxime DU CAMP, Les Ancêtres de la Commune : l'attentat Fieschi, Paris, Charpentier, 1877.

8. COUR DES PAIRS, Affaire des 12 et 13 mai 1839. Rapport fait à la Cour les 11 et 12 juin 1839, par M. Mérilhou, comprenant les faits généraux et la première série de faits particuliers, Paris, Imprimerie Royale, 1839, p. 33-34.

9. Voir sur ce point Michael WALZER, Régicides et révolution, le procès de Louis XVI, Paris, Payot, 1989.

10. Le compte-rendu du procès le plus complet est dans le Journal des débats politiques et littéraires du 8 octobre 1847.

11. Armand BARBES, Deux jours de condamnation à mort, Villelongue d'Aude, Atelier du Gué, [1848], 2005, p. 52.

12. AN, $B^{18} 1397$ Lettre du Procureur général au Garde des Sceaux, 5 octobre 1841.

13. «Du Régicide », Le Moniteur Républicain, $\mathrm{n}^{\circ}$ 6, 16 Floréal An 46, reproduit dans Les Révolutions du XIXe siècle, T. XII, Paris, EDHIS, 1979. 
14. Les ordres du jour saisis dans l'affaire de la rue Pastourelle sont reproduits dans Le Journal des débats politiques et littéraires du 13 décembre 1843.

15. Sur cette affaire voir La Gazette des Tribunaux, $1^{\text {er }}$ et 16 décembre 1836.

16. Il s'agit d'une société parallèle à la Société des Droits de l'Homme et qui prévoyait le recours à l'insurrection pour parvenir à la République. L'appartenance des frères Chaveau, de Husson et des principaux membres du complot à cette société est patente à la lecture des rapports de police. Procès de l'affaire dite complot de Neuilly, devant la cour d'assises de la Seine. Paris, chez Augueux, 1836, p. 3-37.

17. On pourrait ajouter une autre affaire, l'attentat Quenisset, contre la vie des fils de LouisPhilippe qui s'explique également par la désorganisation des sociétés après le 12 mai 1839.

18. Friedrich ENGELS, « Le programme des réfugiés blanquistes de la Commune », Der Volkstaat, 26 juin 1874, reproduit dans Karl Marx, Friedrich Engels, La Commune de 1871, Paris, U.G.E, 10/18, 1971, p. 224-225. Ce texte est avant tout une attaque de circonstance contre un groupe d'exilés français se revendiquant de Blanqui qui quitte l'Internationale après le congrès de La Haye en 1872.

19. On ne dénombre pas moins de six Guillaume Tell et deux variations sur Marino Faliero sur les scènes françaises entre 1828 et 1830.

20. Karelle Vincent a consacré de nombreuses pages à l'analyse des représentations du régicide dans certaines œuvres de Hugo, Musset, Vigny et Balzac: Karelle vinCENT, op.cit., pp. 646-681. Hernani de Victor Hugo est représenté pour la première fois sur la scène du Théâtre français le 25 février 1830. Lorenzaccio de Musset paraît dans la Revue des deux mondes à partir du 23 août 1834.

21. Emmanuel fUREIX, La France des Larmes. Deuils politiques à l'âge romantique (1814-1840), Paris, Champ-Vallon, 2009, p. 455-457.

22. Sur l'exception politique sous la monarchie de Juillet, voir Jean-Claude VIMONT, La prison politique en France. Genèse d'un mode d'incarcération spécifique. XVIIIe-XXe siècles, Paris, Anthropos historiques, 1993, p. 329-338.

23. Cette rumeur se développe après l'attentat de Meunier qui avoue avoir tiré au sort avec des complices la mission de tuer le roi : AN, CC 704 Interrogatoires de Meunier, cités également par Laurent LOUESSARD, op. cit., p. 266.

24. Article du Journal des débats politiques et littéraires du 21 novembre 1841 reproduit dans SaintMarc GIRARDIN, Souvenirs et réflexions d'un journaliste, Paris, Michel Lévy frères, 1859, p. 325.

25. Sur l'influence et la faillite de la phrénologie voir l'étude de Marc RENNEVILLE, Le langage des crânes : histoire de la phrénologie, Paris, Institut d'édition Sanofi-Synthelabo, 2000.

26. Pierre-Antoine BERAUD, De la phrénologie humaine appliquée à la philosophie, aux mours et au socialisme, Paris, Durand, 1848, p. 115.

27. Charles FOURIER, Le Nouveau monde industriel et sociétaire dans CEuvres Complètes, Paris, Librairie sociétaire, 1848, t. VI, p. 69.

28. Le dialogue est repris et développé par le défenseur de Barbès dans son plaidoyer. Voir plus particulièrement dans l'édition républicaine du procès des insurgés : Procès des accusés des 12 et 13 mai devant la Cour des Pairs, Pagnerre, T. I, 1839, p. 241.

29. Jules CLARETIE, Plutarque populaire contemporain illustré. Etudes biographiques, historiques et satiriques sur les hommes du jour, Paris, Librairie centrale, 1870, p. 3.

30. ANONYme [comte d'Alton Shee], "Barbès et Darmès ", Les Agapes, $n^{\circ} 2,14$ décembre 1840, p. 189.

31. L.P. COLLARD DE MARTIGNY, Un mot sur le régicide et spécialement sur l'attentat et le procès de Darmès , Paris, Dellore et Pantin, 1841, p. 5.

32. La journée du 15 mai a fait couler beaucoup d'encre: Piège tendu par les républicains conservateurs aux socialistes pour Henri Guillemin, thèse réfutée par Peter Amann ou Jean-Paul Brunet. Henri GUILLEMIN, 24 février 1848. La première résurrection de la République, Paris, Gallimard, 
1967, p. 325-352. Jean-Paul BRUNET, La police de l'ombre. Indicateurs et provocateurs dans la France contemporaine, Paris, Seuil, 1990, p. 277-284. Peter AMANN, "A Journée in the Making: May 15, 1848 ", The Journal of Modern History, vol. 42, n 1, mars 1970, p. 42-69.

33. Alexis de TOCQUEVILLE, Souvenirs, Paris, Gallimard, [1964], 1999, p. 165

34. Adolphe CHENU, Les conspirateurs, Paris, Garnier frères, 1850. 12 éditions s'épuisent en quelques semaines. Lucien de LA HODDE, Histoire des sociétés secrètes de 1830 à 1848, Paris, JulienLanier, 1850.

35. Les exilés républicains sous le Second Empire sont au cœur de l'ouvrage de Sylvie APRILE, Le Siècle des exilés : bannis et proscrits de 1789 à la Commune, Paris, CNRS Editions, 2010.

36. La Vérité sur l'affaire Orsini par un ancien proscrit, Paris, Publication du nouveau journal républicain, s.d., p. 4.

37. Les historiens sont partagés sur le sujet. Piero Zama penche ainsi pour l'implication de Mazzini dans l'attentat Pianori : Piero ZAMA, Giovanni Pianori contro Napoleone III, Modena, Società tipografica, 1933. En revanche Mazzini n'est pour rien dans l'attentat Orsini mené par des ennemis inconciliables du leader italien.

38. Giuseppe MAZZINI,Letters to Daniel Manin, Londres, 1858, p. 7.

39. Jacques CRETINEAU-joly, Histoire du Sonderbund, Paris, Plon, 1850, T.I, p. 127. Amédée Gabourd, Histoire de Paris depuis les temps les plus reculés jusqu'à nos jours, Paris, Gaume frères, 1865, t. V., p. 139. Sur la rupture entre Mazzini et les socialistes français voir : Jean-Yves FRETIGNE, « Mazzini et les socialistes français: signification et enjeux de la polémique de l'année 1852 ", Revue française d'histoire des idées politiques, $\mathrm{n}^{\circ} 30,2009$, p. 327-358 et Anne-Claire IGNACE, «Giuseppe Mazzini et les démocrates français : débats et reclassements au lendemain du printemps des peuples ", Revue d'histoire du XIX ${ }^{e}$ siècle, n 36, 1, 2008, p. 133-147.

40. Sur l'affaire de l'Opéra Comique, on peut consulter Albert FERME, Les Conspirations sous le Second Empire, Paris, 1869, pp. 19-26. Les dossiers de demande de grâce sont particulièrement instructifs : AN, $\mathrm{BB}^{24}$ 507. Pour le témoignage de Vallès : Jules VALLES, Le Bachelier, in CEuvres, Paris, Gallimard Pléiade, [1881], 1990, t. II, p. 563-565.

41. Voir la correspondance du Procureur général de Lille sur l'affaire : $\mathrm{AN} \mathrm{BB}^{30} 409$.

42. Adrien DANSETTE, L'attentat d'Orsini, Paris, Editions mondiales, 1964.

43. Les Archives Nationales possèdent le dossier de la Cour de cassation sur l'affaire Orsini que ne connaissait pas Adrien Dansette lorsqu'il a rédigé son étude sur Orsini. AN, AM 10 006. Affaire Orsini.

44. BNF, NAF 9583 Papiers Blanqui. Lettre de Blanqui à Rougée, octobre 1853.

45. L'expression est utilisée par Marx dans son compte-rendu des ouvrages de Chenu et Delahodde publié dans la Neue Rheinische Zeitung. Politisch ökonomische Revue, $\mathrm{n}^{\circ} 4$, avril 1850 reproduit dans Karl MARX, Oeuvres, Paris, Gallimard, Pléiade, 1994, t. IV, p. 353.

46. Un résumé de l'affaire qui se termine par une relaxe générale se trouve dans: Auguste SCHELER, Auguste, Annuaire statistique et historique belge, Bruxelles, Kiessling, 1855, p. 239.

47. Emile de GIRARDIN, «Lettre au rédacteur en chef de l'Indépendance belge, 3 août 1857 », dans L'Empire avec la liberté, Paris, Michel Lévy, 1859, p. 15.

48. Parmi les plus violents, citons : Félix PYAT, Lettre à une balle, s.l., s.d., [1859].

49. Sur cet auteur important du romantisme social, voir: Guy SABATIER, Le mélodrame de la République sociale et le théâtre de Félix Pyat, Paris, L'Harmattan, 1998, 2 vol.

50. Félix PYAT, Lettre au Parlement et à la presse à propos de l'attentat d'Orsini contre Napoléon III, Londres, Imprimerie Universelle, 1858, p. 14.

51. Rapport du Procureur général Mongis du 8 avril 1859, cité dans Jean-Claude FARCY, Les Rapports des procureurs généraux de la Cour d'appel de Dijon (décembre 1849-juillet 1870), Dijon, Editions Universitaires de Dijon, 2003, p. 346.

52. Mémoires d'un parisien : la gloriole, Paris, Victor Havard, 1888, p. 241. 


\section{RÉSUMÉS}

L'attentat est un mot du pouvoir au XIXe siècle. Le terme met l'accent sur la gravité d'une attaque contre l'ordre politique et social sans plus de précisions quant au moyen employé. L'attentat par excellence est alors celui qui vise la personne du chef de l'Etat qui, même sans être sacré, reste la clé de voûte des systèmes politiques de la Monarchie de Juillet ou du Second Empire. Le pouvoir suspecte systématiquement le complot derrière l'attentat. De fait, sous la Monarchie de Juillet et au début du Second Empire les oppositions révolutionnaires se constituent en sociétés secrètes et se posent en concurrentes de l'Etat. Pour ces conspirateurs, le recours à l'attentat, est souvent contre-productif: le meurtre politique est considéré comme moins efficace et moins légitime que l'insurrection populaire pour s'emparer du pouvoir. Conspirateurs et forces de l'ordre se livrent une bataille politique dont les enjeux symboliques ne sont pas les moindres. Après le 15 mai 1848, la figure du conspirateur est décrédibilisée. La répression impitoyable qui s'abat sur les sociétés secrètes à la fin de la République et sous le Second Empire accroît leur impuissance. L'attentat contre le souverain, rendu plus destructeur par la technologie, devient alors une arme par défaut mais aussi un instrument de vengeance contre le coup d'état et les plébiscites favorables au régime. Le regard porté sur l'attentat change : il acquiert même une certaine reconnaissance politique comme l'atteste le traitement réservé à Orsini en 1858. Après 1870, l'attentat à l'explosif est identifié comme un moyen d'action des révolutionnaires dans la société française.

In France, in the $19^{\text {th }}$ century, the term 'attentat' only belonged to the vocabulary of the authorities. It emphasized the gravity of attacks against the political and social order, whatever may be the means. The quintessential 'attentat' was the attack against the monarch, who sacred or not, was still the keystone of the political arch during the July Monarchy and the Second French Empire. The police were systematically suspecting a plot behind regicide attempts. Under the July Monarchy until the beginning of the Second French Empire, underground opposition groups emerged and tried to impose themselves as an alternative to the regime. The 'attentat' was often counterproductive for those conspirators: to seize power, political murders were considered less efficient and legitimate than popular insurrections. The conspirators and the government were engaged in a political battle, entailing strong symbolic stakes. After the journée of 15 May 1848, the figure of the conspirator was discredited. Secret societies were severly repressed and could not lead insurrections anymore. Attacks against the monarch, more devastating with the use of explosives, became not only a weapon by default but also an instrument of revenge against the man of the coup d'état and those in favour of the regime. Bomb attacks were then perceived differently: they even acquired some political recognition as the treatment reserved to Orsini in 1858 demonstrates. After 1870, bomb attacks were linked to the revolutionaries in people's minds.

\section{INDEX}

Mots-clés : conspiration, régicides, sociétés secrètes, imaginaire politique

Keywords : conspiracy, regicides, secret societies, political imaginary 


\section{AUTEUR}

\section{JEAN-NOËL TARDY}

Docteur en histoire, Université Paris-1 Panthéon-Sorbonne, Centre d'Histoire du $19^{\mathrm{e}}$ siècle 